\title{
Characterization of Conjugated Polymer Poly(fluorene-co-thiophene) and Its Application as Photosensitizer of $\mathrm{TiO}_{2}$
}

\author{
Rongliang Qiu, ${ }^{1}$ Lin Song, ${ }^{1}$ Dongdong Zhang, ${ }^{1}$ Yueqi Mo, ${ }^{2}$ Eric Brewer, ${ }^{3}$ and Xiongfei Huang ${ }^{1}$ \\ ${ }^{1}$ School of Environmental Science and Engineering, Sun Yat-Sen University, Guangzhou 510275, China \\ ${ }^{2}$ College of Material Science and Engineering, South China University of Technology, Guangzhou 510641, China \\ ${ }^{3}$ Viridian Environmental, LLC, Arlington, VA 22207, USA
}

Correspondence should be addressed to Rongliang Qiu, eesqrl@mail.sysu.edu.cn

Received 26 February 2007; Accepted 15 April 2007

Recommended by M. Sabry A. Abdel-Mottaleb

\begin{abstract}
The copolymer poly(fluorene-co-thiophene) (PFT) has strong absorption in the visible light region. In this paper, PFTs with four different thiophene contents were evaluated for their suitability as photosensitizers of $\mathrm{TiO}_{2}$. All four of the PFTs were capable of being excited by light with wavelengths above $455 \mathrm{~nm}$, and all had reductive potentials more negative than the conduction band potential of $\mathrm{TiO}_{2}$. Consequently, it was deemed that transfer of electrons from the excited PFTs to the conduction band of TiO was thermodynamically possible. PFTs with higher thiophene content had more electron transitions in the excited state, resulting in increased photocatalytic activity of PFT-sensitized $\mathrm{TiO}_{2}$. The method used to prepare the combined $\mathrm{PFT} / \mathrm{TiO} \mathrm{O}_{2}$ photocatalyst affected its photocatalytic activity. Best results in this study were achieved by dropwise addition of a tetrahydrofuran solution of PFT to a stirred $\mathrm{TiO}_{2}$ /ethanol suspension.
\end{abstract}

Copyright (c) 2008 Rongliang Qiu et al. This is an open access article distributed under the Creative Commons Attribution License, which permits unrestricted use, distribution, and reproduction in any medium, provided the original work is properly cited.

\section{INTRODUCTION}

Photosensitization of the semiconducting oxide $\mathrm{TiO}_{2}$ is a method for extending its photoresponse in the visible light region by physically combining it with another semiconductor that acts as a photosensitizer. As p-type semiconductors, photosensitizers, which are commonly organic dyes, have strong absorption in the visible light region and can inject electrons into the conduction band of $\mathrm{TiO}_{2}$ when excited by visible light, giving rise to a series of photochemical processes and allowing the photocatalytic reactions mediated by $\mathrm{TiO}_{2}$ to operate under visible light irradiation.

Conjugated polymers composed of repeated small aryl ring molecules such as benzene, thiophene, and pyrrole are other types of organic semiconductors. Although the energy level difference between $\pi$-bonding and antibonding orbits in a single aryl ring is large, it is found that as the number of repeated units increases, $\pi$ orbits overlap each other, causing the energy level difference between $\pi$-conjugated bonding and antibonding orbits to decrease sharply and fall eventually to less than $4 \mathrm{eV}$. Energy level differences of this mag- nitude make these polymers suitable for use as semiconductors. In addition, some of these polymers have a number of optical properties, such as electroluminescence and nonlinear optical behavior, that make them especially useful as photovoltaic materials. At present, conjugated polymers such as poly(3-methylthiophene) [1], poly(phenylene vinylene) [2], polyaniline [3], polypyrrole [4], poly(o-methoxy aniline) [5], poly(3-undecyl-2,2' -bithiophene) [6], and poly[2(3-thienyl)-ethoxy-4-butylsulfonate] (PTEBs) [7] are being increasingly used in the field of photovoltaics as solid electrolytes and electrode sensitizers [8].

Methods for sensitizing semiconductors are similar to those for sensitizing electrodes of solar energy cells. However, little work has been done to date on the use of conjugated polymer-sensitized semiconductors to photocatalyze the degradation of organics under visible light irradiation, in part because of limitations of the polymers themselves. One problem is that the HOMO energy level of polymers is generally higher than those of inorganic materials and dyes, so polymers tend to be unstable and easily oxidized. In addition, the electron mobility of polymers is relatively poor. Finally, 
it can be difficult to physically combine polymers and inorganic semiconductors, because most polymers are not soluble in water or ethanol. However, as research in this area continues, more and more polymers with improved characteristics are being synthesized. For example, the wide band gap polymer polyfluorene (PF) and the narrow band gap polymer polythiophene have improved antioxidative ability. Moreover, electron transfer takes place easily in thiophenebased polymers.

In this paper, a series of conjugated copolymers containing fluorene and thiophene [9] were investigated to evaluate their suitability as photosensitizers of $\mathrm{TiO}_{2}$.

\section{EXPERIMENTAL}

\subsection{Materials}

A series of copolymer PFTs with thiophene contents of $5 \%$, $15 \%, 30 \%$, and 50\% (so called PFT5, PFT15, PFT30, and PFT50, resp.) were provided by College of Material Science and Engineering, South China University of Technology; the chemical structure is presented in Figure 1, $\mathrm{TiO}_{2}$ (Degussa P25: $80 \%$ anatase, $20 \%$ rutile; BET $\sim 50 \mathrm{~m}^{2} / \mathrm{g}$ ). Purified water was obtained from a Millipore filtration system (Millipore ZLXS50020). All other chemicals were analytical grade and used without further purification.

\subsection{Measurements and procedures}

An HP 8453 diode array UV-Vis spectrophotometer (Agilent, USA) was used to directly record the UV-Vis spectra of the copolymer PFTs using chloroform as solvent. The electrochemical properties of PFTs were obtained with a CHI 660 A electrochemical workstation. Phenol concentration was monitored by UV-2450 spectrophotometer (Shimadzu, Japan) at a wavelength of $510 \mathrm{~nm}$.

Sensitization of $\mathrm{TiO}_{2}$ was performed according to the literature [10]. The photocatalytic degradation experiment was carried out by adding $50 \mathrm{mg}$ photocatalyst into $50 \mathrm{~mL}$ phenol aqueous solution with $10 \mathrm{mg} / \mathrm{L}$ initial concentration. A $250 \mathrm{~W} \mathrm{GaI}_{3}$ lamp (DJG250, Shanghai Hualun Lighting Co. Ltd., China) with maximum radiant wavelength matched to the absorption wavelength of PFT was used as the visible light source. UV and IR cutoff filters were placed between the lamp and the glass reactor to ensure irradiation by visible light (400-700 nm) only. Light intensity was measured by a UV-A ultraviolet meter equipped with two probes with wavelengths of $420 \mathrm{~nm}$ and $365 \mathrm{~nm}$, respectively. The glass reactor was open to air to ensure enough oxygen in the reaction solution. Prior to irradiation, the solution containing photocatalyst and phenol was magnetically stirred in darkness for 30 minutes to establish adsorption-desorption equilibrium. After a specified interval of irradiation time, a $5 \mathrm{~mL}$ aliquot of the reaction mixture was centrifuged and filtered through a $0.22 \mu \mathrm{m}$ filter (Millipore), and the change in the concentration of phenol was monitored spectrophotometrically.

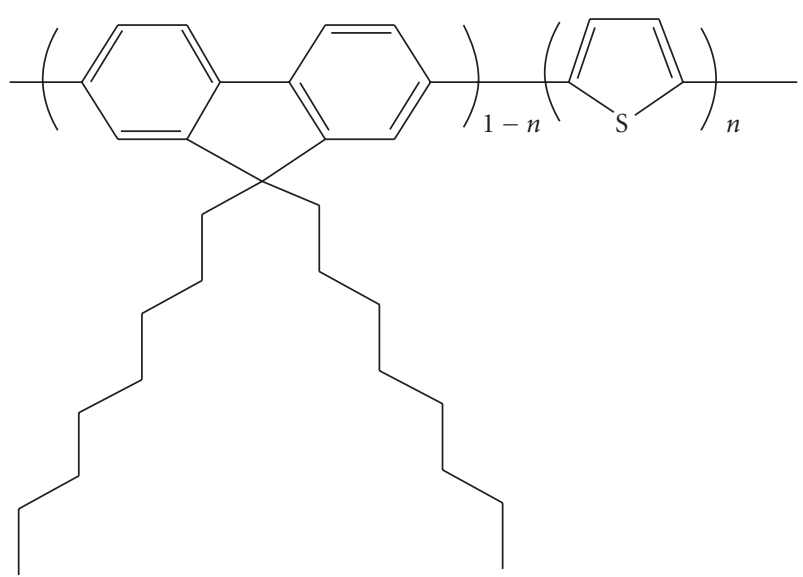

FIGURE 1: Structure of conjugated polymer poly(fluorene-cothiophene).

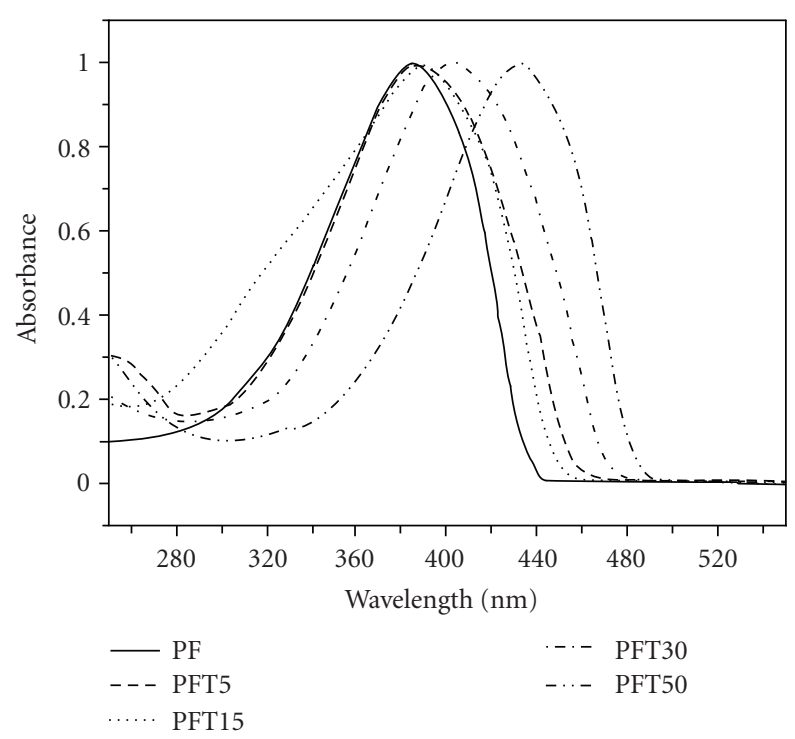

FIGURE 2: UV-Vis spectra of the different copolymers.

\section{RESULTS AND DISCUSSION}

\subsection{Electrochemical properties of PFTs}

Having strong absorption in the visible light region is the most important property of a photosensitizer. Figure 2 shows the UV-Vis spectra of the copolymers PF, PFT5, PFT15, PFT30, and PFT50 in chloroform. These polymers all have strong absorption in the visible light region. As expected, the intensity and maximum absorption peaks progressively increased and were red-shifted with increasing content of thiophene in the PFTs. The maximum absorption peaks are at $384 \mathrm{~nm}, 384 \mathrm{~nm}, 390 \mathrm{~nm}, 400 \mathrm{~nm}$, and $433 \mathrm{~nm}$, respectively. From Figure 2 it is observed that the absorption peaks of PFT5 and PFT15 were little changed from that of PF, while their spectra were slightly red-shifted toward the visible light region. The absorption peak of PFT30 was redshifted from $384 \mathrm{~nm}(\mathrm{PF})$ to $400 \mathrm{~nm}$, and its absorption edge 
TABLE 1: Wavelength at peak absorption and electrochemical properties of PFTs.

\begin{tabular}{l|cccccc}
\hline Copolymers & $\begin{array}{c}\lambda_{\text {absmax }} \\
(\mathrm{nm})\end{array}$ & $\begin{array}{c}E_{\mathrm{ox}} \\
(\mathrm{V})\end{array}$ & $\begin{array}{c}E_{\mathrm{red}} \\
(\mathrm{V})\end{array}$ & $\begin{array}{c}E_{g} \\
(\mathrm{eV})\end{array}$ & $\begin{array}{c}E_{\mathrm{HOMO}} \\
(\mathrm{eV})\end{array}$ & $\begin{array}{c}E_{\mathrm{LUMO}} \\
(\mathrm{eV})\end{array}$ \\
\hline PTF5 & 455 & 1.38 & -1.35 & 2.73 & -5.78 & -3.05 \\
PTF15 & 470 & 1.31 & -1.33 & 2.64 & -5.71 & -3.07 \\
PTF30 & 480 & 1.30 & -1.28 & 2.58 & -5.70 & -3.12 \\
PTF50 & 490 & 1.22 & -1.31 & 2.53 & -5.62 & -3.09 \\
\hline
\end{tabular}

was red-shifted to $480 \mathrm{~nm}$. In the case of PFT50, the red shifting was more obvious; the absorption peak and edge were red-shifted to $433 \mathrm{~nm}$ and $490 \mathrm{~nm}$, respectively. The increased red shifting that was associated with increased thiophene content of the PFTs was due to strong absorption of visible light by the thiophene units of the polymer. Compared with PF oligomers, the blocks of spatial configuration between thiophene ring and benzene ring in PFTs are smaller than those between benzene rings in PF, and twists between thiophene ring and benzene ring take place more easily, leading to higher coplanarity and a larger conjugated system in PFTs. In addition, the lone pair electrons of the sulfur atom in thiophene units increase the electron cloud density in the PFT chain, thereby increasing its ability to act as an electron donor and decreasing its band gap. Thus, higher thiophene content of a PFT is associated with higher coplanarity, lower band gap, and red shifting of its absorption spectrum.

The second most important property of a photosensitizer of $\mathrm{TiO}_{2}$ is that its energy level matches that of $\mathrm{TiO}_{2}$. Only if the reductive potential of the photosensitizer is more negative than the energy level of the conduction band of $\mathrm{TiO}_{2}$, it is thermodynamically possible for excited electrons to be injected from the conduction band of the photosensitizer to the conduction band of $\mathrm{TiO}_{2}$.

Table 1 presents the wavelength at peak absorption and electrochemical properties of the PFTs used in this study. The peak and onset of absorption wavelength of PFTs were obtained from Figure 2, and the band gaps $\left(E_{g}\right)$ were estimated by (1) [11]:

$$
\begin{aligned}
E_{g} & =1240 / \lambda_{\text {onset }}, \\
E_{\text {red }} & =\left(E_{g} E_{\text {ox }}\right), \\
E_{\mathrm{HOMO}} & =e\left(E_{\mathrm{OX}}+4.4\right),(\mathrm{eV}) \\
E_{\mathrm{LUMO}} & =e\left(E_{\mathrm{red}}+4.4\right),(\mathrm{eV}) .
\end{aligned}
$$

All oxidative potentials $\left(E_{\mathrm{ox}}\right)$ of PFTs were measured by a CHI 660 A electrochemical workstation, using a platinum electrode as working electrode, a saturated calomel electrode (SCE) as reference electrode, and a platinum wire as assistant electrode in $0.1 \mathrm{~mol} / \mathrm{L}$ of tetra- $n$-butylammonium tetrafluoroborate $\left(\mathrm{Bu}_{4} \mathrm{NPF}_{6}\right)$ in anhydrous acetonitrile as a supporting electrolyte with a scan rate of $50 \mathrm{mV} / \mathrm{s}$. The reductive potentials $\left(E_{\text {red }}\right), \mathrm{HOMO}$, and LOMO energy levels were calculated by empirical equations (2)-(4) [12], respectively.

The results in Table 1 indicate that the band gaps of these PFTs were less than or equal to $2.73 \mathrm{eV}$, which corresponds

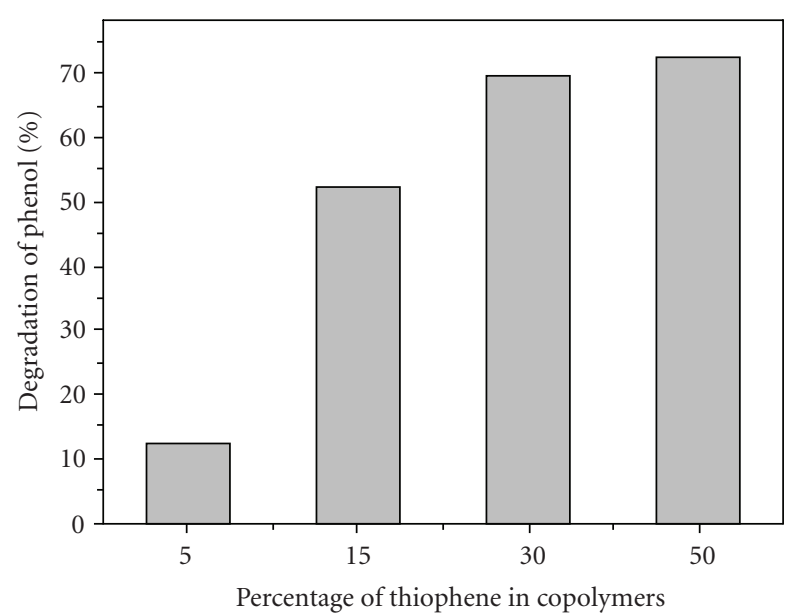

Figure 3: Photodegradation of phenol using PFT-sensitized $\mathrm{TiO}_{2}$ $[\text { phenol }]_{i}=10 \mathrm{mg} / \mathrm{L},[$ photocatalyst $]=1 \mathrm{~g} / \mathrm{L}$, irradiation time $=10$ hours.

to excitation by wavelengths at or above $455 \mathrm{~nm}$. The reductive potentials were approximately $-1.30 \mathrm{~V}$ and were all more negative than the energy level of the conduction band of $\mathrm{TiO}_{2}$. Thus, transfer of electrons from excited PFTs to the conduction band of $\mathrm{TiO}_{2}$ is thermodynamically possible. It was also found that with increasing thiophene content in the main chain of the polymer, the oxidative potential gradually decreased from $1.38 \mathrm{~V}$ (for PFT5) to $1.22 \mathrm{~V}$ (for PFT50). These oxidative potentials are higher than those of many polymers, and lead to stronger antioxidative ability.

\subsection{Photodegradation of phenol using PFT-sensitized $\mathrm{TiO}_{2}$}

The photodegradation of phenol by PFT-sensitized $\mathrm{TiO}_{2}$ is presented in Figure 3. During the photodegradation experiment, the light intensity was $E_{420}=11.1 \times 100 \mu \mathrm{w} / \mathrm{cm}^{2}$ and $E_{365}=1.4 \mu \mathrm{w} / \mathrm{cm}^{2}$. The maximum absorption wavelength of phenol is about $270 \mathrm{~nm}$ and phenol has no response for visible light. Our earlier experimental results showed that no phenol decomposes under visible light irradiation. From Figure 3, all of the PFTs were able to photosensitize $\mathrm{TiO}_{2}$. The photocatalytic activity of sensitized $\mathrm{TiO}_{2}$ excited by visible light increased with increasing thiophene content of the PFT. The relatively small increase in catalytic activity between PFT30- and PFT50-sensitized $\mathrm{TiO}_{2}$ was attributed to two factors. First, addition of 50\% thiophene reduced the molecular weight of the PFTs from above 20000 to about 6000 . As a result, there was less entwisting between PFT chains, so, the polymer film was weaker and did not adsorb as well onto the $\mathrm{TiO}_{2}$ powder. Second, it was observed that PFT50 had lower solubility in tetrahydrofuran (THF) than the other polymers, which interfered with the physical process of combining it with the $\mathrm{TiO}_{2}$. Thus, we used PFT30 as sensitizer in the following experiments.

Commonly, PF has a larger band gap than PFT, with maximum absorption in the UV region [13, 14]. With increasing thiophene content in the main chain of PF, its band 
gap decreases and its absorption spectrum is gradually redshifted. It was found that PFT with a higher thiophene content had more electron transitions when excited by visible light, which resulted in greater photocatalytic activity of PFT-sensitized $\mathrm{TiO}_{2}$. Hara and coworkers [15] reported for the first time a new kind of sensitizers based on introducing thiophene moieties into a coumarin backbone, and then bonded it to the surface of $\mathrm{TiO}_{2}$ with $-\mathrm{COOH}$ bonds. Using this new compound in dye-sensitized solar cells, they achieved a remarkable solar energy-to-electricity conversion efficiencies of up to $7.7 \%$ under simulated solar irradiation $\left(100 \mathrm{~mW} \cdot \mathrm{cm}^{-2}\right)$, which was the highest efficiency obtained among solar cells based on organic dye photosensitizers in the past decades. Luo and coworkers [16], studying the properties of oligothiophene carboxylic acid sensitized $\mathrm{TiO}_{2}$ films, found that oligothiophene carboxylic acid could be used as a cosensitizer with $\mathrm{N} 3\left[\mathrm{Ru}\right.$ complex, $\mathrm{Ru}(\mathrm{II}) \mathrm{L}_{2}(\mathrm{SCN})_{2}$, $\mathrm{L}=2,2^{\prime}$-bipyridyl-4, 4' -dicarboxylate] dye, extending its spectrum and improving its photoelectric properties. Thiophene has also been used to improve the properties of porphyrin photosensitizers $[17,18]$. Ha and coworkers [19] investigated singlet oxygen generation efficiencies of porphyrin and thiophene-modified porphyrin using time-resolved and steady-state spectroscopic methods. They found that the introduction of the sulfur atom from thiophene into the porphyrin core greatly increased the intersystem crossing rate due to spin-orbit coupling enhancement, resulting in enhanced triplet and singlet oxygen quantum yields. These results suggested that thiophene can improve the photophysical efficiency of photosensitizers. From the above, it is clear that addition of thiophene directly affects the properties of combinative photosensitizers.

Additionally, PFTs showed higher stability than other small molecular dyes such as rhodamine $\mathrm{B}$ and eosin $\mathrm{Y}$ under irradiation because of the higher oxidative potentials. Reused after 10 hours irradiation, PFT30 still had efficient photosensitization to $\mathrm{TiO}_{2}$, and the photocatalytic activity of PFT30sensitized $\mathrm{TiO}_{2}$ slightly decreased less than $10 \%$ under the same experiments conditions.

\subsection{Effect of photosensitization method on photodegradation of phenol}

In studies of organic pollution degradation using dyesensitized $\mathrm{TiO}_{2}$, the primary methods used for combining dye with $\mathrm{TiO}_{2}$ are simple adsorption $[20,21]$, chemical bonding [22], and LB (Langmuir-Blodgett) film technology [23]. The combining of $\mathrm{TiO}_{2}$ with polymers depends primarily on adsorption and wrapping by polymer long chains. The conjugated polymer PFTs used in this paper have high solubility in aromatic solvents, begin to precipitate in ethanol, and have very low solubility in water. Thus, in the combining method that we designed, PFTs were first dissolved in THF, a good solvent, and then the solution was added to ethanol, a worse solvent, in order to initiate a gradual precipitation of PFT via wrapping of long chains. Finally, the solvents were removed by a rotary evaporator (RE-100, QUICKFIT, England), leaving the PFT coated on the surface of the $\mathrm{TiO}_{2}$. The precipitation of PFT and its bonding to $\mathrm{TiO}_{2}$ are af-

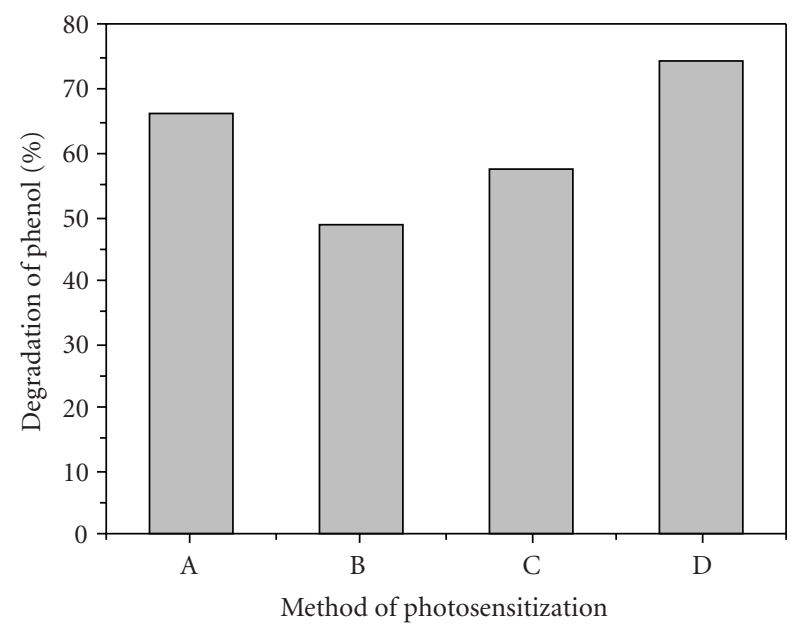

Figure 4: Photodegradation of phenol using PFT30-sensitized $\mathrm{TiO}_{2}$ with different methods of photosensitization. A: PFT30/THF solution added to $\mathrm{TiO}_{2}$ /ethanol suspension quickly. $\mathrm{B}$ : $\mathrm{TiO}_{2} /$ ethanol suspension added to PFT30/THF solution quickly. C: PFT30/THF solution added to $\mathrm{TiO}_{2}$ /ethanol suspension in two parts. D: PFT30/THF solution added dropwise to $\mathrm{TiO}_{2}$ /ethanol suspension.

fected by the physical parameters of the combining process, including the method of mixing the solvents, the mixing time, and the method of adding the $\mathrm{TiO}_{2}$ powder. These parameters must be optimized in order to avoid uneven deposition of PFT or premature agglomeration of $\mathrm{TiO}_{2}$, both of which can adversely affect configuration and separateness of the combinative photocatalyst and impair its photocatalytic activity. Figure 4 shows the results of an experiment in which the photodegradation of phenol was catalyzed by PFT30-sensitized $\mathrm{TiO}_{2}$ prepared using four different methods. The photodegradation experiments conditions were the same with Section 3.2 in this paper.

Figure 4 indicates that addition of PFT30/THF solution to a $\mathrm{TiO}_{2}$ /ethanol suspension produced a more active photocatalyst than addition of $\mathrm{TiO}_{2} /$ ethanol suspension to the PFT30/THF solution. The best results were obtained by dropwise addition of PFT30/THF solution to the $\mathrm{TiO}_{2}$ /ethanol suspension, which ensured that the PFT precipitated slowly enough to evenly coat the surface of the $\mathrm{TiO}_{2}$.

\section{CONCLUSION}

Four copolymers, consisting of poly(fluorene-co-thiophene) (PFT) with a range of thiophene content, were evaluated as photosensitizers of $\mathrm{TiO}_{2}$. All of the PFTs had strong absorption in the visible light region and had band gaps less than or equal to $2.73 \mathrm{eV}$, which corresponds to excitation by wavelengths above $455 \mathrm{~nm}$. The reductive potentials of the PFT copolymers were approximately $-1.30 \mathrm{~V}$, which was more negative than the energy level of the $\mathrm{TiO}_{2}$ conduction band. Thus, transfer of electrons from the excited PFTs to the conduction band of $\mathrm{TiO}_{2}$ is thermodynamically possible. All of the PFTs were capable of photosensitizing $\mathrm{TiO}_{2}$. Photocatalytic activity of PFT-sensitized $\mathrm{TiO}_{2}$ increased with 
increasing thiophene content of the PFT. The method of preparation of the combined $\mathrm{PFT} / \mathrm{TiO}_{2}$ photocatalyst affected its photocatalytic activity. The best method of photocatalyst preparation in our study was dropwise addition of a solution of PFT in tetrahydrofuran to a stirred suspension of $\mathrm{TiO}_{2}$ in ethanol.

\section{ACKNOWLEDGMENT}

This work was financed by the New Century Excellent Talent Program of MOE (NCET-04-0790), China.

\section{REFERENCES}

[1] A. F. Nogueira, L. Micaroni, W. A. Gazotti, and M.-A. de Paoli, "Enhanced photoresponse of poly(3-methylthiophene) supported on $\mathrm{TiO}_{2}$," Electrochemistry Communications, vol. 1, no. 7, pp. 262-265, 1999.

[2] T. J. Savenije, J. M. Warman, and A. Goossens, "Visible light sensitization of titanium dioxide using a phenylene vinylene polymer," Chemical Physics Letters, vol. 287, no. 1-2, pp. 148153, 1998.

[3] M. S. Liu, Y. Z. Hao, X. B. Qiao, M. Z. Yang, M. S. Cai, and Y. F. Li, "Photoelectrochemical studies on nanoporous $\mathrm{TiO}_{2} / \mathrm{Pan}$ film electrode," Electrochemistry, vol. 4, no. 3, pp. 246-251, 1998.

[4] M. Martini and M.-A. de Paoli, "Effect of the electrolyte cations and anions on the photocurrent of dodecylsulphate doped polypyrrole films," Solar Energy Materials and Solar Cells, vol. 73, no. 3, pp. 235-247, 2002.

[5] A. F. Nogueira, N. Alonso-Vante, and M.-A. de Paoli, "Solidstate photoelectrochemical device using poly(o-methoxy aniline) as sensitizer and an ionic conductive elastomer as electrolyte," Synthetic Metals, vol. 105, no. 1, pp. 23-27, 1999.

[6] C. D. Grant, A. M. Schwartzberg, G. P. Smestad, J. Kowalik, L. M. Tolbert, and J. Z. Zhang, "Characterization of nanocrystalline and thin film $\mathrm{TiO}_{2}$ solar cells with poly(3-undecyl-2,2' bithiophene) as a sensitizer and hole conductor," Journal of Electroanalytical Chemistry, vol. 522, no. 1, pp. 40-48, 2002.

[7] J. T. McLeskey Jr. and Q. Qiao, "Hybrid solar cells from water-soluble polymers," International Journal of Photoenergy, vol. 2006, Article ID 20951, 6 pages, 2006.

[8] J. N. de Freitas, V. C. Nogueira, B. I. Ito, et al., "Dye-sensitized solar cells and solar module using polymer electrolytes: stability and performance investigations," International Journal of Photoenergy, vol. 2006, Article ID 75483, 6 pages, 2006.

[9] Q. Hou, Y. Niu, W. Yang, R. Yang, M. Yuan, and Y. Cao, “Synthesis and electroluminescent properties of copolymer of fluorene and thiophene," Acta Polymerica Sinica, no. 2, pp. 161165, 2003.

[10] L. Song, R. Qiu, Y. Mo, D. Zhang, H. Wei, and Y. Xiong, "Photodegradation of phenol in a polymer-modified $\mathrm{TiO}_{2}$ semiconductor particulate system under the irradiation of visible light," Catalysis Communications, vol. 8, no. 3, pp. 429-433, 2007.

[11] T. Sasaki, N. Koshizaki, J.-W. Yoon, and K. M. Beck, "Preparation of $\mathrm{Pt} / \mathrm{TiO}_{2}$ nanocomposite thin films by pulsed laser deposition and their photoelectrochemical behaviors," Journal of Photochemistry and Photobiology A: Chemistry, vol. 145, no. 12, pp. 11-16, 2001.
[12] D. M. de Leeuw, M. M. J. Simenon, A. R. Brown, and R. E. F. Einerhand, "Stability of n-type doped conducting polymers and consequences for polymeric microelectronic devices," Synthetic Metals, vol. 87, no. 1, pp. 53-59, 1997.

[13] Q. Pei and Y. Yang, "Efficient photoluminescence and electroluminescence from a soluble polyfluorene," Journal of the American Chemical Society, vol. 118, no. 31, pp. 7416-7417, 1996.

[14] Y. W. Di, X. W. Ba, D. M. Xie, and R. S. Wang, "The novel functional materials polyalkylfluorene derivatives and their applications," Journal of Functional Materials, vol. 28, no. 4, pp. 346-349, 1997.

[15] K. Hara, M. Kurashige, Y. Dan-Oh, et al., "Design of new coumarin dyes having thiophene moieties for highly efficient organic-dye-sensitized solar cells," New Journal of Chemistry, vol. 27, no. 5, pp. 783-785, 2003.

[16] Z. Luo, S.-X. Tan, J. Zhai, et al., "Photo-electric conversion properties of oligo-thiophene carboxylic acid sensitized porous $\mathrm{TiO}_{2}$ films," Chemical Journal of Chinese Universities, vol. 25, no. 1, pp. 154-156, 2004.

[17] C.-H. Hung, C.-K. Ou, G.-L. Lee, and S.-M. Peng, "Structure and characterization of the first metal complex of dithiaporphyrin: $\mathrm{Ru}\left(\mathrm{S}_{2} \mathrm{TTP}\right) \mathrm{CI}_{2}$," Inorganic Chemistry, vol. 40, no. 26, pp. 6845-6847, 2001.

[18] L. Latos-Grazyński, J. Lisowski, M. M. Olmstead, and A. L. Balch, "21-Thiatetra-p-tolylporphyrin and its copper(II) bicarbonate complex. Structural effects of copper-thiophene binding," Journal of the American Chemical Society, vol. 109, no. 14, pp. 4428-4429, 1987.

[19] J.-H. Ha, S. Ko, C.-H. Lee, W.-Y. Lee, and Y.-R. Kim, "Effect of core atom modification on photophysical properties and singlet oxygen generation efficiencies: tetraphenylporphyrin analogues core-modified by oxygen and/or sulfur," Chemical Physics Letters, vol. 349, no. 3-4, pp. 271-278, 2001.

[20] D. Chatterjee and A. Mahata, "Demineralization of organic pollutants on the dye modified $\mathrm{TiO}_{2}$ semiconductor particulate system using visible light," Applied Catalysis B: Environmental, vol. 33, no. 2, pp. 119-125, 2001.

[21] D. Chatterjee and A. Mahata, "Visible light induced photodegradation of organic pollutants on dye adsorbed $\mathrm{TiO}_{2}$ surface," Journal of Photochemistry and Photobiology A: Chemistry, vol. 153, no. 1-3, pp. 199-204, 2002.

[22] X. Li, W. Zhao, and J. Zhao, "Visible light-sensitized semiconductor photocatalytic degradation of 2, 4-dichlorophenol," Science in China, Series B: Chemistry, vol. 45, no. 4, pp. 421425, 2002.

[23] X.-S. Feng, S.-Z. Kang, H.-G. Liu, and J. Mu, "Study of the photophysical properties of composite film assembled of porphyrin and $\mathrm{TiO}_{2}$ nanoparticles," Thin Solid Films, vol. 352, no. 1-2, pp. 223-227, 1999. 


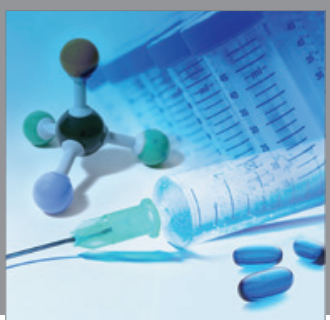

International Journal of

Medicinal Chemistry

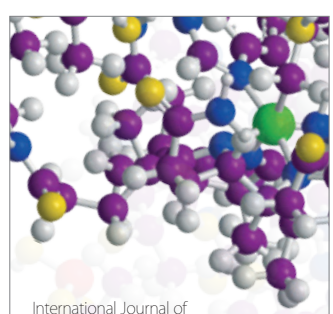

Carbohydrate Chemistry

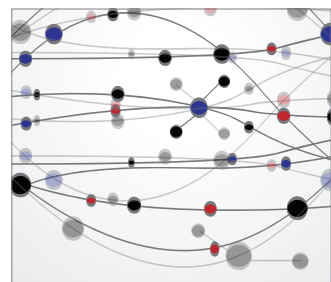

The Scientific World Journal
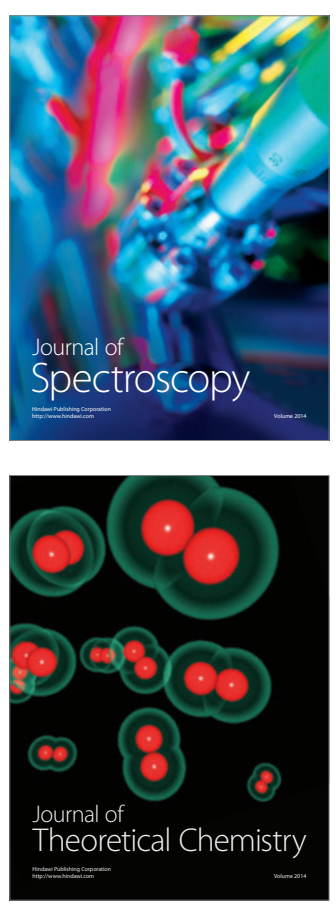
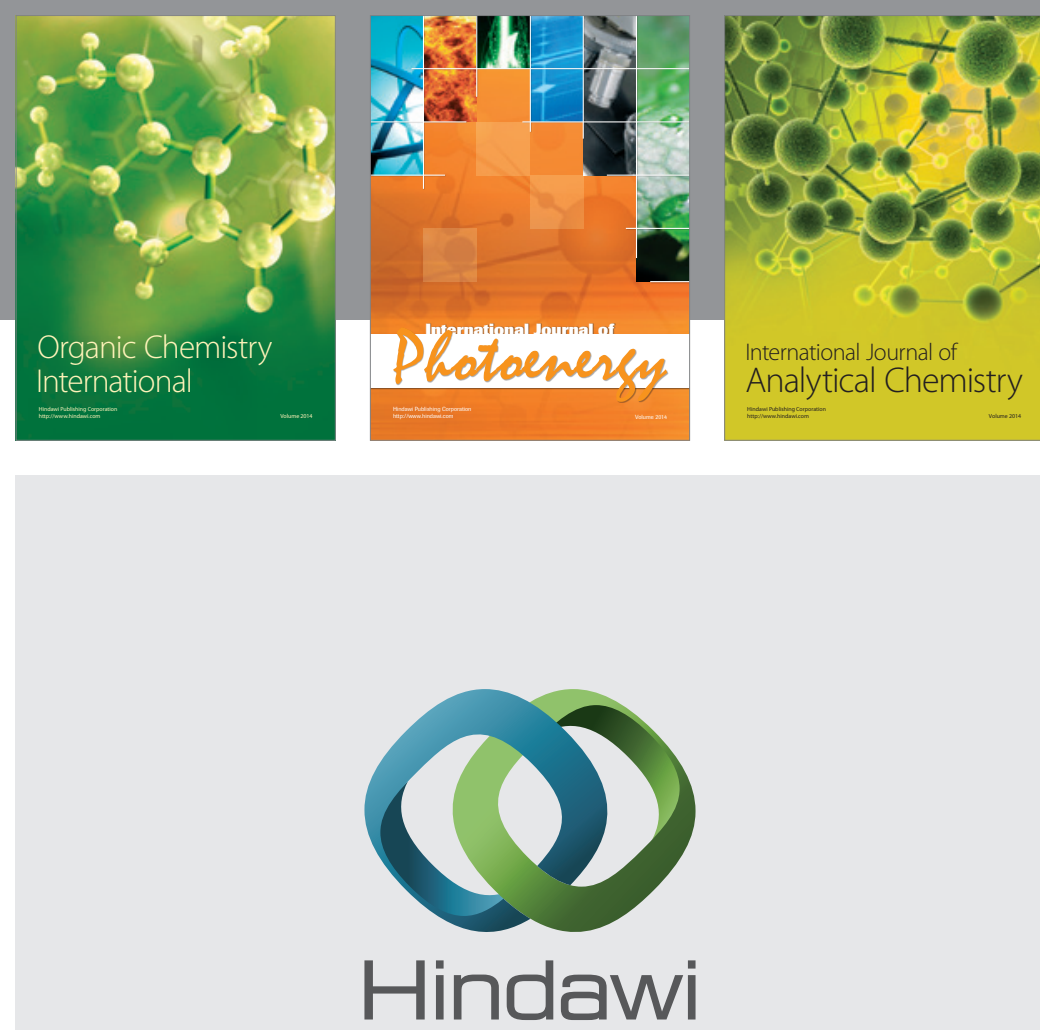

Submit your manuscripts at

http://www.hindawi.com
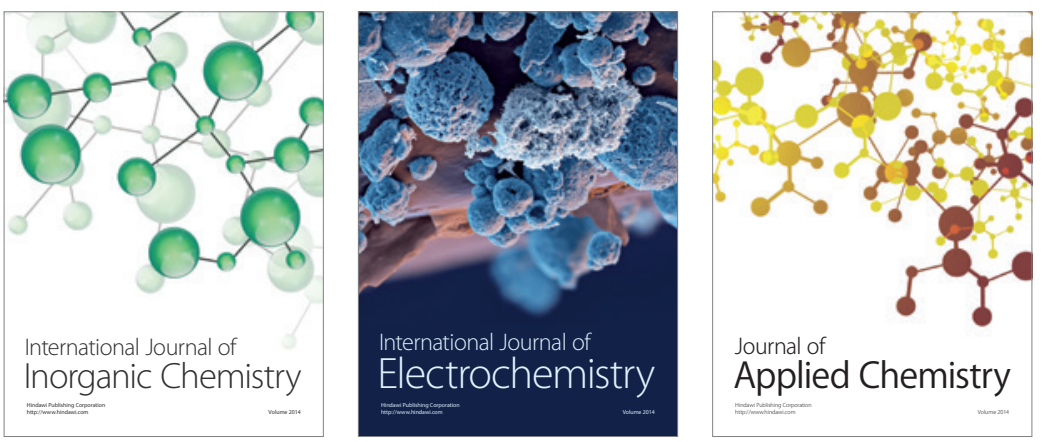

Journal of

Applied Chemistry
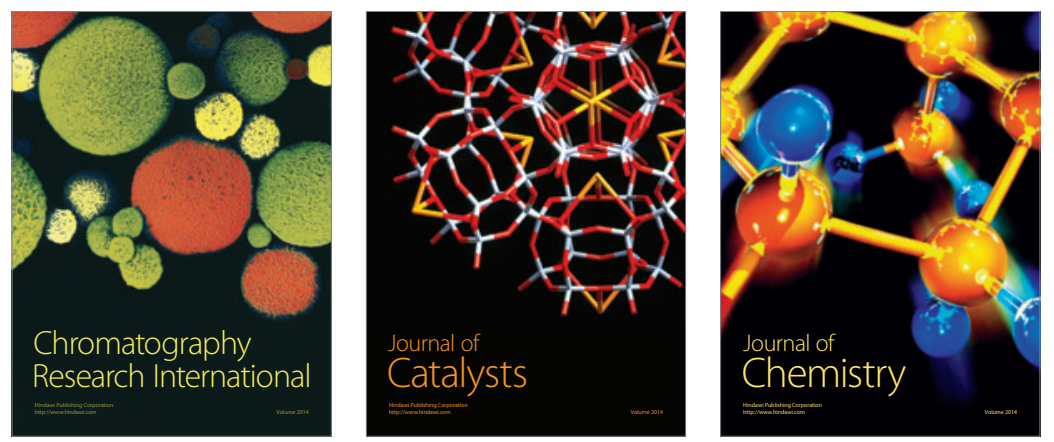
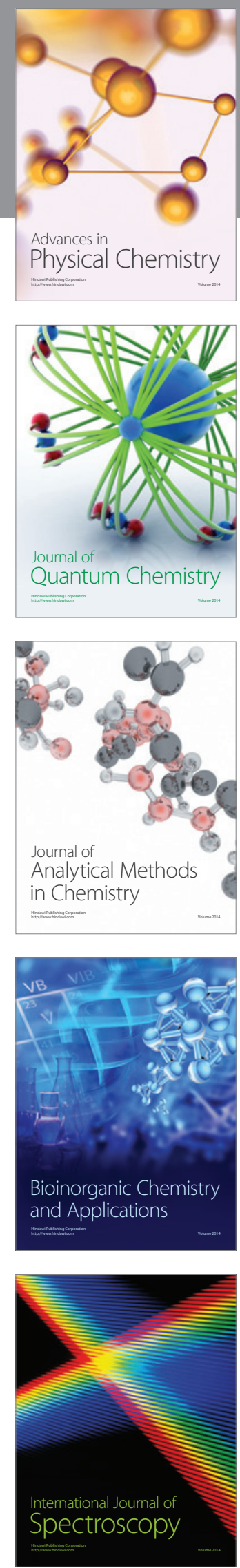